\section{S10.2 CERVICAL CANCER SCREENING: BENEFITS AND CHALLENGES OF HPV SELF-SAMPLING IN MOZAMBIQUE}

N Osman*. Eduardo Mondlane University, Maputo, Mozambique

10.1136/sextrans-2021-sti.46

Mozambique has one of the highest burdens of cervical cancer in the world, with an incidence rate of 42.8 and mortality rate of 35.7 per 100000 women. There is a lack of population based organized screening programs, and few doctors and nurses trained to diagnose and treat cervical abnormalities and invasive cancer. The HIV prevalence, a significant risk factor for cervical cancer, is $12.6 \%$ nationally. Cervical cancer screening has been introduced in the country using visual inspection with acetic acid (VIA) followed by immediate treatment with cryotherapy or referral for the loop electrosurgical excision procedure (LEEP) if the cervical lesion(s) do not meet the criteria for cryotherapy. However, uptake has been limited and it is estimated that $<5 \%$ of women have undergone screening. Furthermore, many screen positive women are lost to followup and are not receiving treatment. Most women in Mozambique therefore present with advanced stage cervical cancer and the majority die of their disease.

In order to overcome these challenges, we are performing studies using human papillomavirus (HPV) testing for cervical screening. We recently completed a study of 898 women who underwent provider collected HPV testing. We noted a HPV positivity rate of $23.7 \%$. Women living with HIV were twice as likely to test positive for HPV as HIV negative women (39.2\% vs 19.9\%, p<0.001). Most HPV positive women (194 of $213,91.1 \%$ ) completed all steps of their diagnostic workup and treatment, which included cryotherapy $(n=158$, $77.5 \%)$, loop electrosurgical excision procedure $(n=30$, $14.7 \%$ ), or referral to a gynecologist or gynecologic oncologist $(n=5,2.5 \%)$. Based on these results, we are now performing a follow-up study of HPV-testing using self-collected cervicovaginal swabs. To date, we have enrolled 2,076 of 14,600 women. We are evaluating accuracy as well as both patient and provider satisfaction with self-sampling as part of the study.

\section{S10.3 POINT-OF-CARE HPV TESTING AND TREATMENT FOR CERVICAL CANCER SCREENING AND ELIMINATION IN PAPUA NEW GUINEA}

1,2A Vallely*. 'Kirby Institute, UNSW Sydney, Sydney, Australia; ${ }^{2}$ Papua New Guinea Institute of Medical Research, Goroka, Papua New Guinea

10.1136/sextrans-2021-sti.47

Papua New Guinea (PNG) has among the highest estimated burdens of cervical cancer globally, but lacks national programs for cervical screening or human papillomavirus (HPV) vaccination. Following the disappointing performance of visual inspection of the cervix with acetic acid (VIA) for cervical screening in this setting, we evaluated a novel same-day 'testand-treat' screening model based on point-of-care HPV-DNA testing of self-collected vaginal specimens followed by sameday thermal ablation of the cervix. Self-collected specimens tested at point-of-care on the GeneXpert platform (Xpert HPV; Cepheid, Sunnyvale, CA) had comparable performance to laboratory-based assays both for the detection of high-risk oncogenic HPV types and for detection of underlying pre-cancer and cancer. This strategy was highly acceptable among women and health care workers; highly cost-effective compared to screening based on VIA or Pap test cytology; and with suitable training and support, feasible to implement in routine primary care facilities in this setting. We further showed that if $70 \%$ of women aged 30-54 years were screened twice in their lifetime as recommended by WHO, this point-of-care 'test and treat' model could reduce both cervical cancer incidence and mortality in PNG by more than $50 \%$. This approach represents a promising new model for cervical screening in PNG and other high-burden, low-income countries embarking on a path towards cervical cancer elimination.

\section{Oral presentations}

\section{Basic science for bacterial STIs}

\subsection{IDENTIFICATION AND FUNCTIONAL CHARACTERIZATION OF ANTIMICROBIAL PEPTIDES FROM TREPONEMA PALLIDUM}

S Houston*, E Schovanek, K Conway, S Mustafa, A Gomez, R Ramaswamy, A Haimour, M Boulanger, L Reynolds, C Cameron. University of Victoria, Victoria, Canada

\subsection{6/sextrans-2021-sti.48}

Background Antimicrobial peptides (AMPs) are low molecular weight proteins involved in pathogen elimination. Bacteria, in particular Gram-positive commensals, have been shown to produce AMPs to inhibit and kill competing microbes. The observations that Treponema pallidum can survive and establish infection within polymicrobial sites that are abundant in commensals and/or pathogens, and $\sim 6-9 \%$ of the T. pallidum proteome is predicted to be composed of 'miniproteins' $(\leq 150$ amino acids in size) of unknown function provided a rationale for investigating whether these small $\mathrm{T}$. pallidum proteins function as AMPs.

Methods A bioinformatics pipeline comprised of six AMP prediction servers was developed for identifying potential treponemal AMPs and their critical core regions (AMPCCRs) in T. pallidum miniproteins of unknown function. Selected AMPCCR candidates were chemically synthesized and assessed for antimicrobial activity against a panel of biologically and clinically relevant bacterial species using broth microdilution and a modified agar dilution method.

Results Four potential AMPCCRs (Tp0451a_N, Tp0451a_C, Tp0749_N and Tp0749_C) exhibited bacteriostatic and bactericidal activity (MIC and MBC ranges of $1.0-256 \mu \mathrm{g} / \mathrm{ml}$ ) against Escherichia coli (Tp0749_C), Pseudomonas aeruginosa (Tp0749_C), Streptococcus pyogenes (Tp0451a_C), Mycobacterium species (Tp0451a_N, Tp0451a_C, Tp0749 N, Tp0749_C), and Neisseria gonorrhoeae (Tp0451a_C).

Conclusion Our findings are consistent with the novel concept that AMP production is an important, previously undiscovered mechanism that may contribute to survival of $\mathrm{T}$. pallidum within the host. These investigations have established proof-ofconcept for our AMP discovery bioinformatics pipeline via the experimental identification of the first AMPs from T. pallidum. This novel research approach has the potential to reveal an important survival mechanism that is more widespread in pathogenic bacteria than current data suggest. 